\title{
Queensland tick typhus (Rickettsia australis) in a man after hiking in rural Queensland
}

Stephen A Thomas, Jason Wu

CASE

A man aged 51 years presented with a one-week history of fever, lethargy, anorexia and generalised arthralgias, with an erythematous eruption (Figure 1) and a large plaque with eschar noted on examination (Figure 2). The patient was afebrile and haemodynamically stable, with an otherwise unremarkable examination and no organomegaly or lymphadenopathy.

Further questioning revealed a history of visiting the Bunya Mountains National Park, Queensland, for a hiking trip two weeks prior to presentation. The patient was not aware of being bitten by ticks or other insects; however, Queensland tick typhus was suspected clinically, given this is a common presentation in patients with tick bites who have visited that area, while Sweet syndrome was considered as a remote differential. Initial management included doxycycline $100 \mathrm{mg}$ twice daily and follow-up.

Lesional biopsy for histopathology was performed at presentation, confirming a mononuclear vasculitis consistent with rickettsial infection, and no features of Sweet syndrome. Initial serology immunofluorescence assay was negative ( $<1: 128$ titre) for Rickettsia rickettsii (the serological test that is cross-reactive for Rickettsia australis). Other blood tests, including liver and renal function tests, were normal.

At review two weeks later, the patient reported that most of his symptoms had resolved and demonstrated clearing of his rash. Some ongoing minor lethargy persisted. Rickettsial serology was repeated at this time (four weeks after likely bite exposure) and showed an elevated $R$. rickettsii titre.

\section{Question 1}

Where and how do people contract Queensland tick typhus?

\section{Question 2}

How does the disease typically present?

\section{Question 3}

What testing is done for this disease?

\section{Question 4}

What is the treatment for this disease?

\section{Answer 1}

Rickettsia refers to a group of Gramnegative bacterial infections. Australian tick typhus, otherwise known as Queensland tick typhus (R. australis), is a commonly encountered rickettsial infection in Australia. The name, Queensland tick typhus, arose after soldiers training in Queensland during World War II were affected. ${ }^{1}$ R. australis may occur from tick exposure, typically along the east coast of Australia (Wilsons Promontory in Victoria to tropical north Queensland; Figure 3). Infection can result from the bite of an infected tick or from exposure to the faeces of infected hosts. Ticks in the affected areas generally survive by feeding off other wildlife (Figure 4), including marsupials and rodents.

\section{Answer 2}

There is an incubation period of two to 14 days from the time of bite. Initial clinical presentation typically includes eschar at the bite site, fever, headache and a confluent erythematous eruption. If left untreated for more than one to two weeks, the disease poses some risk of pneumonitis, encephalitis, septic shock or death. ${ }^{2}$ Prolonged lethargy or fatigue, even after rash clearance, is a common symptom reported with rickettsial infection. Blood tests may reveal an elevated white cell count, acute transaminitis and elevated C-reactive protein.

\section{Answer 3}

Serological R. rickettsii indirect immunofluorescence assay detects the infection at 1:128 dilution, with crossreactivity between $R$. rickettsii (Rocky Mountain spotted fever) and $R$. australis (Queensland tick typhus). ${ }^{3}$ Positive cases that are detected at 1:128 dilution have repeated titrations of sera, providing a final titre result. ${ }^{4}$

Serology takes at least six days before obtaining a positive result and is currently the gold standard for testing. ${ }^{4}$ Initial negative serological studies do not rule out rickettsial infection and should not alter treatment completion in potentially infected patients. Testing could subsequently be repeated in more suspicious cases four weeks after 


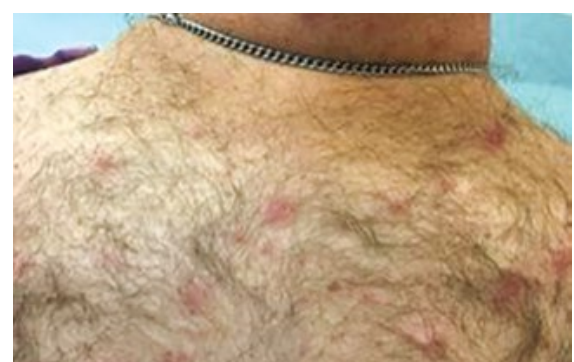

Figure 1. Initial clinical presentation of non-confluent erythematous plaques on upper back

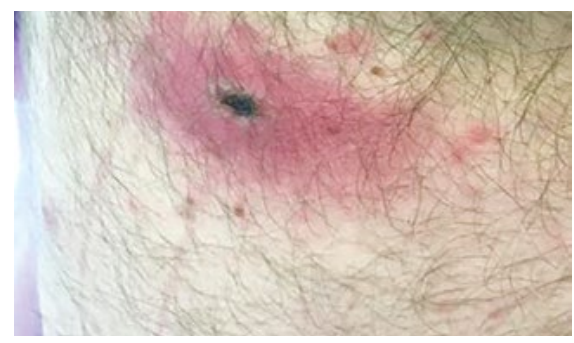

Figure 2. Initial clinical presentation of central eschar on broad erythematous plaque on back (suggestive of original bite site)

suspected exposure but is unlikely to alter management.

An alternative diagnostic method by polymerase chain reaction (PCR) is available nationally from the Australian Rickettsial Reference Laboratory. PCR looks for rickettsial DNA, although this has inferior diagnostic sensitivity. PCR of a skin biopsy or swab from an eschar lesion, or from the patient's blood, may be more sensitive during days one to five of the infection. ${ }^{4,5}$

\section{Answer 4}

Initial management of suspected cases should include a seven-day course of oral doxycycline $100 \mathrm{mg}$ twice daily. If allergy or contraindications to doxycycline exist, oral azithromycin $250 \mathrm{mg}$ may be used once daily for seven days. Treatment response is usually rapid.

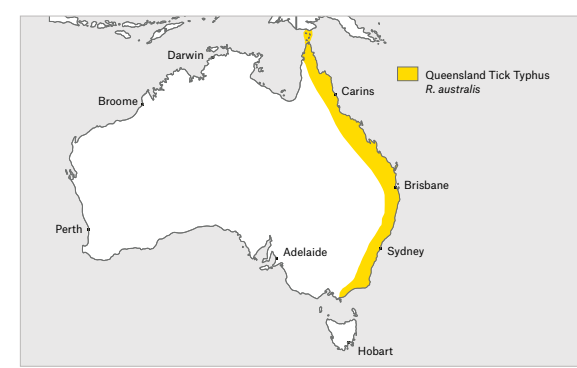

Figure 3. Distribution map of Queensland Tick Typhus in Australia. ${ }^{4}$

Adapted with permission from Graves S. Update on Australian rickettsial infections. Surry Hills, NSW: Australasian Society for Infectious Diseases, 2017

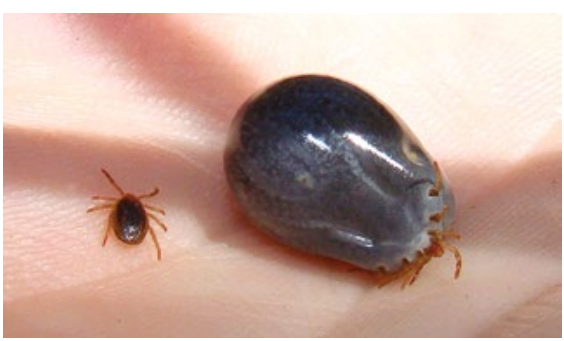

Figure 4. Photograph of an unfed and fed tick. 'Tick before and after feeding' by Bjørn Christian Tørrissen, www.pvv.org/ bct/nsw/imagepages/ image53.htm. Licence at https://creativecommons. org/licenses/by-sa/3.0/

\section{Key points}

- R. australis is an infection typically presenting with eschar at bite site, fevers, lethargy and arthralgias in patients who have visited endemic areas (typically rural or bush areas along the eastern seaboard of Australia).

- Clinical presentations of rickettsial infection in the context of recent exposure to endemic areas should cause the physician to have a low threshold for treatment, with or without patient knowledge of a tick bite.

- Serological R. rickettsii immunofluorescence is the current gold standard for testing; however, typically, this will not test positive until at least one week following tick bite exposure. PCR is a more appropriate test on days one to five after bite exposure but has inferior overall sensitivity.

\section{Authors}

Stephen A Thomas MBBS, Department of Dermatology, Princess Alexandra Hospital, Brisbane, Qld. stephenanthonythomas@hotmail.com

Jason Wu FACD, Department of Dermatology, Princess Alexandra Hospital, Brisbane, Qld

Competing interests: None.

Provenance and peer review: Not commissioned, externally peer reviewed.

\section{References}

1. Appuhamy RD, Tent J, Mackenzie JS. Toponymous diseases of Australia. Med J Aust 2010;193(11-12):642-46.

2. Expert Group for Antibiotic. Rickettsial infections. In: eTG complete [Internet]. Melbourne: Therapeutic Guidelines Limited, 2014.

3. health.vic. Rickettsial infections. Melbourne: Department of Health and Human Services, 2017. Available at www2.health.vic.gov.au/public-health/ infectious-diseases/disease-information-advice/ rickettsial-infection [Accessed 11 August 2017].

4. Graves S. Update on Australian rickettsial infections. Surry Hills, NSW: Australasian Society for Infectious Diseases, 2017. Available at www. asid.net.au/documents/item/415 [Accessed 11 August 2017].

5. Wang JM, Hudson BJ, Watts MR, et al. Diagnosis of Queensland tick typhus and African tick bite fever by PCR of lesion swabs. Emerg Infect Dis 2009;15(6):963-65. doi: 10.3201/eid1506.080855. 